\title{
Introducing Calculus to the High School Curriculum: Curves, Branches and Functions
}

Dr. Andrew Grossfield P. E., Vaughn College of Aeronautics \& Technology

Throughout his career Dr. Grossfield, has combined an interest in engineering and mathematics. He earned a BSEE at the City College of New York. During the early sixties, he obtained an M.S. degree in mathematics at the Courant Institute of NYU at night while working full time as an engineer for aerospace/avionics companies. He studied continuum mechanics in the doctoral program at the University of Arizona. He is a member of ASEE, IEEE and MAA. 


\title{
Introducing Calculus to the High School Curriculum: Curves, Branches and Functions
}

\begin{abstract}
The number of high school students taking calculus and AP calculus is increasing, and this trend appears to be accelerating. Examining this phenomenon leads to some questions: Is the memorization required by most students to pass calculus healthy for the individual student? Is training a citizenry to memorize calculus facts psychologically healthy for American society as a whole? There has been criticism of various aspects of conventional algebra and calculus texts which present barriers to the understanding of the concept of continuous smooth functions. Such barriers include lack of focus, lack of structure, unmotivated definitions, the clutter of applications, the number of pages in the texts and the emphasis on proofs at the expense of true insight and the essential needs of our society for a citizenry capable of understanding mathematical concepts.
\end{abstract}

In our society calculus has had a reputation for being difficult to master. If this subject is introduced to a much wider high school audience in the same way it has been taught in colleges, it might turn out to deter more students from entering the Science, Technology, Engineering and Math (STEM) disciplines. However maintaining our national technological leadership requires that STEM enrollments increase and that more of our nation's young citizens acquire deeper insight into mathematical and statistical thinking. Although calculus has traditionally been taught with an emphasis on proofs, it does not mean that the best interests of society are served by continuing and extending to a larger audience this underperforming process. Alternative approaches must be considered.

Basically, the concepts of calculus are neither abstract nor overly difficult. However, unlike calculus, the concepts of algebra are less obvious, and more students have to struggle harder in order to understand essential algebraic concepts. Moreover, it is crucial that students acquire some dexterity in algebraic form changing manipulations and solving procedures, preferably before starting their study of calculus.

This, the first in a set of three papers, is planned to provide the concepts of pre-calculus visually and intuitively in order to reveal the intrinsic ultimate simplicity of calculus and spare a student from having to read the entire 500 pages of conceptually cluttered verbose, disorganized conventional text in order to acquire an overview. My hopes are that by providing a focus of study, specifically algebraic and transcendental curves, and by providing intuitive and visual definitions, while maintaining an organized topic structure and by delaying the proofs, we can create a conceptual environment where more students and teachers will gain insight relatively quickly into the nature of calculus. The plan is to interpret the concepts of calculus either geometrically in terms of continuous curves or algebraically as one variable continuously controlling a second variable. Subsequently a student, enabled with the goals and structure of the course in calculus, can refer to conventional texts to fill in and expand on subordinate details. 


\section{Introduction - What is Calculus?}

Historically the ancient Greeks understood how to compute the perimeters and areas of general polygons, figures composed of straight line segments. As for curves, they knew that both the area and the perimeter of a circle were related to the diameter by a single number, $\pi$, about which much remained to be discovered. They could compute the lengths of the circular arcs and the area of an ellipse, but could not compute the circumference of an ellipse or arc lengths and areas for general closed curves. First, rules and identities of algebra had to be developed. In addition, the idea of Descartes was necessary: to plot variables on orthogonal axes which would enable equations in two variables to be interpreted as curves. Once that path was cleared, Newton and Leibniz could discover the rules which would enable the computation of arc lengths and areas from the rules for computing the directions of tangent lines to the curves.

Essentially, algebra and calculus comprise the study of piecewise continuous, smooth curves. Piecewise continuous means the curves can have jumps or gaps at only a few separate points. Piecewise smooth means the curves have a tangent line everywhere except at a few separate points.

Usually, students learn in high school algebra the different kinds and characteristics of curves; that is, they learn to graph simple curves, to find the zeros of polynomials and rational curves and to solve for the intersections of simple curves. It is also important that students learn to visualize the curve which is associated with a particular equation.

As an example, examine the graph of the fourth degree polynomial, $y=x^{4}-2 x^{2}+.2 x+1$, which is shown below in Figure 1.

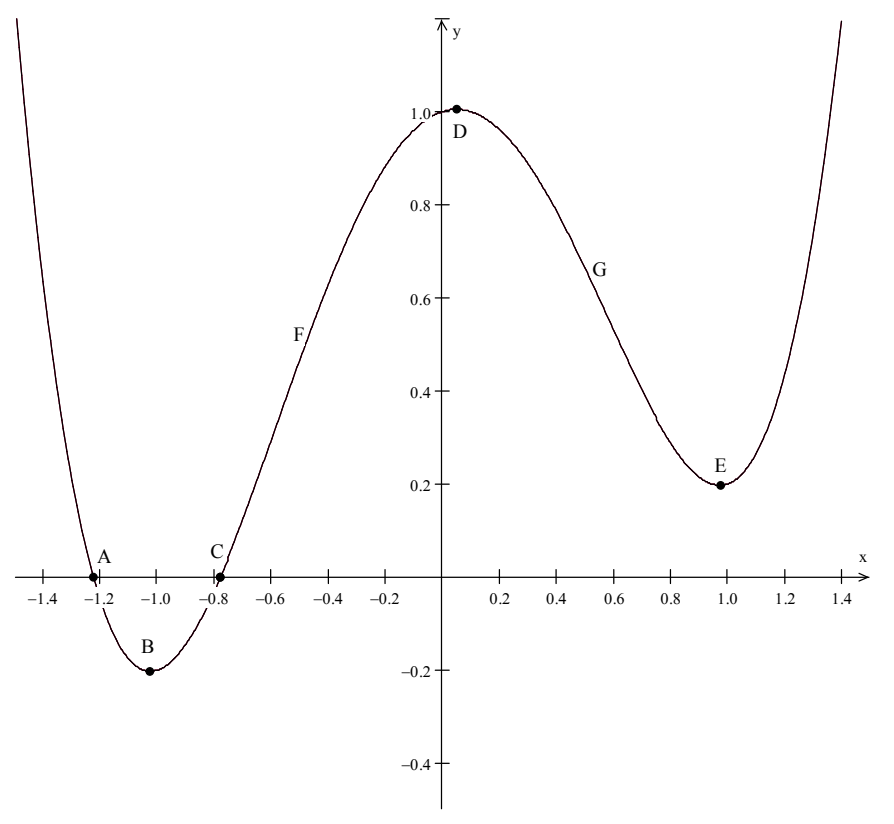

Figure 1 A fourth degree polynomial: $y=x^{4}-2 x^{2}+.2 x+1$ 
The graph indicates:

(1) The extent of the curve, horizontally and vertically

(2) the position of $y$ for each corresponding $x$,

(3) the points which are peaks and valleys of $y$

(4) the alternating intervals of $x$ between the peaks and valleys where the curve is either rising and falling and the alternating intervals where the curve is either turning up or turning down.

Horizontally, this curve extends infinitely to the left and to the right. Vertically, the curve extends upward to infinity both in the first and second quadrants. The curve has two minimum points at $\mathrm{B}$ and $\mathrm{E}$ and the vertical values never go lower than about -0.02 . The curve has a local maximum at $\mathrm{D}$.

The curve has two zeroes at about $\mathrm{x}=-1.22$ and $\mathrm{x}=-0.78$. The $\mathrm{y}$-values are negative for the horizontal coordinates in the interval between $\mathrm{A}$ and $\mathrm{C}$, that is $-1.22<\mathrm{x}<-0.78$. Otherwise the vertical values are positive.

A student might wonder how such a curve with similar features could be constructed. The effect of squaring a polynomial can be observed in figure 2 where the graph of the square of the parabola $y=x^{2}-1$ is plotted. The following principles should be noted and can be used.

1) Performing any smooth continuous operation such as squaring on a smooth continuous curve produces a smooth continuous curve.

2) The square will be non-negative.

3) If the original curve is either evenly or oddly symmetric about the y-axis then the square will be evenly symmetric.

4) The horizontal locations of zeros and extrema remain unchanged.

5) Where the original curve has a negative minimum, the square will have positive maximum.

6) Squaring changes the zeros into minimums of value zero.

7) The square of any polynomial produces a non-negative polynomial of twice the degree.

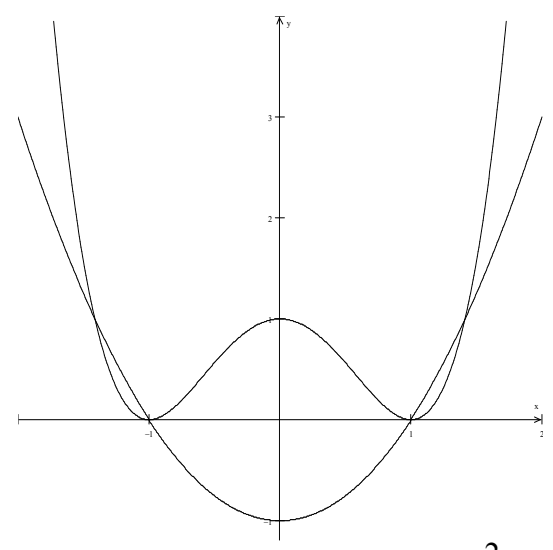

Figure 2 Squaring a parabola: $\mathrm{y}=\mathrm{x}^{2}-1$ and $\mathrm{y}=\left(\mathrm{x}^{2}-1\right)^{2}$ 
The asymmetry is produced by adding the straight line $\mathrm{y}=0.2 \mathrm{x}$ which has the effect of raising curve in the right half plane and lowering the curve in the left half plane. Adding the straight line has also affects the locations of all the extreme points. The double zero in the right half plane has disappeared and the double zero in the left half plane has become two separate zeros. It can be observed in Equation 1 that the effect of the successive operations identically equals the curve graphed in Figure 1.

$$
\left(x^{2}-1\right)^{2}+0.2 x=x^{4}-2 x^{2}+1+0.2 x \quad \text { Equation } 1
$$

Imagine for a moment, a particle moving along the curve. At each point, the particle has a position, specified by its coordinates. Additionally, if at each point the curve has a tangent line, that is, if the curve has no corners, then the motion has a unique direction specified by the slope of the tangent line. A corner, which is technically called a cusp, is a sharp change in direction. And lastly, if the curve is not a straight line, then the curve may either lay above the tangent line (called concave or turning up which occurs at points A and E in Figure 1) or below (called concave or turning down, which occurs at point D in Figure 1).

Conceptually, calculus can be divided into two subjects:

1) differential calculus, which concerns with the computation of the tangent lines to curves and the computations of 1) position, 2) direction and 3) turning at each point and

2) integral calculus whose concern is the computation of arc lengths, areas, volumes and other properties which require the same or slightly modified techniques of computation.

The process in differential calculus of computing the direction of the tangent lines is called differentiation. The process in integral calculus of finding areas and arc lengths is called integration. Calculus as a whole can then be said to consist of the study of differentiation and integration and the properties of curves.

\section{The uses of letters in algebra - Unknowns}

One kind of equation with one letter and numbers is called a conditional equation. The letter represents a number which currently may be unknown. A conventional algebraic problem is to apply the laws of algebra to change the form of the conditional equation to the form, $\mathrm{x}=\mathrm{N}$, where $\mathrm{N}$, the solution of the equation, represents the unknown value or values, if any. This process of successively changing the forms of a conditional equation in order to reveal the unknown values is called solving the equation for $\mathrm{x}$.

If the condition equation was $x^{3}+3=30$, the equation could be changed to $x^{3}=27$ and then to $\mathrm{x}=27^{1 / 3}=3$. A check verifies that indeed $3^{3}+3=30$ and the solution is $\mathrm{x}=3$.

\section{Quantitative laws - variables}

Another use for letters in algebra is as part of a notational system for writing quantitative laws. As an example, consider the problem of relating the area of a rectangle to the measurements of its sides. The law could be written as an English sentence or, as scientists have discovered, 
measurements of the length and width can be represented with the symbols, $\mathrm{L}$ and $\mathrm{W}$, and the value of the area can be represented with the symbol, A. The law can then be described by the equation $\mathrm{A}=\mathrm{L}^{*} \mathrm{~W}$. Note that at the moment, none of the values of $\mathrm{L}, \mathrm{W}$ or $\mathrm{A}$ may be known but what is known is that when any two of the three values are provided, the equation can then be used to determine the value represented by the remaining symbol. The letters used in this way to represent the quantitative values in the laws are called variables.

Quantitative laws are found in every study where quantities are counted, measured or computed. Examples from arithmetic include the commutative law, $a b=b a$, and the distributive law, $\mathrm{c}(\mathrm{a}+\mathrm{b})=\mathrm{ca}+\mathrm{cb}$. Examples from geometry include the various formulas for area, and the Pythagorean Theorem. And examples from physics include Newton's law, F = ma, and Einstein's mass-energy equivalence $\mathrm{E}=\mathrm{mc}^{2}$. Imagine how these laws would be described if letters could not be used! A good exercise for a student is to try to write one of these laws in ordinary English prose.

The success of algebraic techniques in advancing scientific progress can be attributed to the ability to write complicated relationships compactly as equations and the ability to manipulate equations to change forms and find solutions. Consider as an example, Newton's law of motion, which is known to be $\mathrm{F}=\mathrm{ma}$. Then the rules of algebra dictate that both of the equations $\mathrm{a}=\mathrm{F} / \mathrm{m}$ and $\mathrm{m}=\mathrm{F} / \mathrm{a}$ are true, assuming neither of the denominators equals zero.

Writing the laws using variable notation has advantages. The equation in variables is more compact than the statement in words, which makes the equation easier to scan and comprehend. And in addition, the equation can be manipulated using the rules of algebra. In an equation with several variables, if it is possible to solve for one of the variables uniquely in terms of the others, say F, as in Newton's law, F = ma, then F is said to be a function of $\mathrm{m}$ and $\mathrm{a}$.

An algebraic equation in two variables, like $\mathrm{x}$ and $\mathrm{y}$, can be represented as a multi-valued curve in the 2-dimensional Cartesian $\mathrm{x}-\mathrm{y}$ plane. This equation does not by itself determine a value for either of the variables, $x$ or $y$, both of which can have a range of values. But if one of the variables, say $\mathrm{x}$, is given a value, then the equation becomes a conditional equation for the other variable, $y$. Solving this condition for $y$, may yield a set of values, $y_{i}$, which correspond to the given value of $\mathrm{x}$. Then the pairs $\left(\mathrm{x}, \mathrm{y}_{1}\right),\left(\mathrm{x}, \mathrm{y}_{2}\right)\left(\mathrm{x}, \mathrm{y}_{3}\right)$ etc. can be plotted. As an example, consider the graph of a circle, shown below in Figure 3. If many values of $x$ are chosen close enough to one another the outline of the curve described by the equation can be observed. 


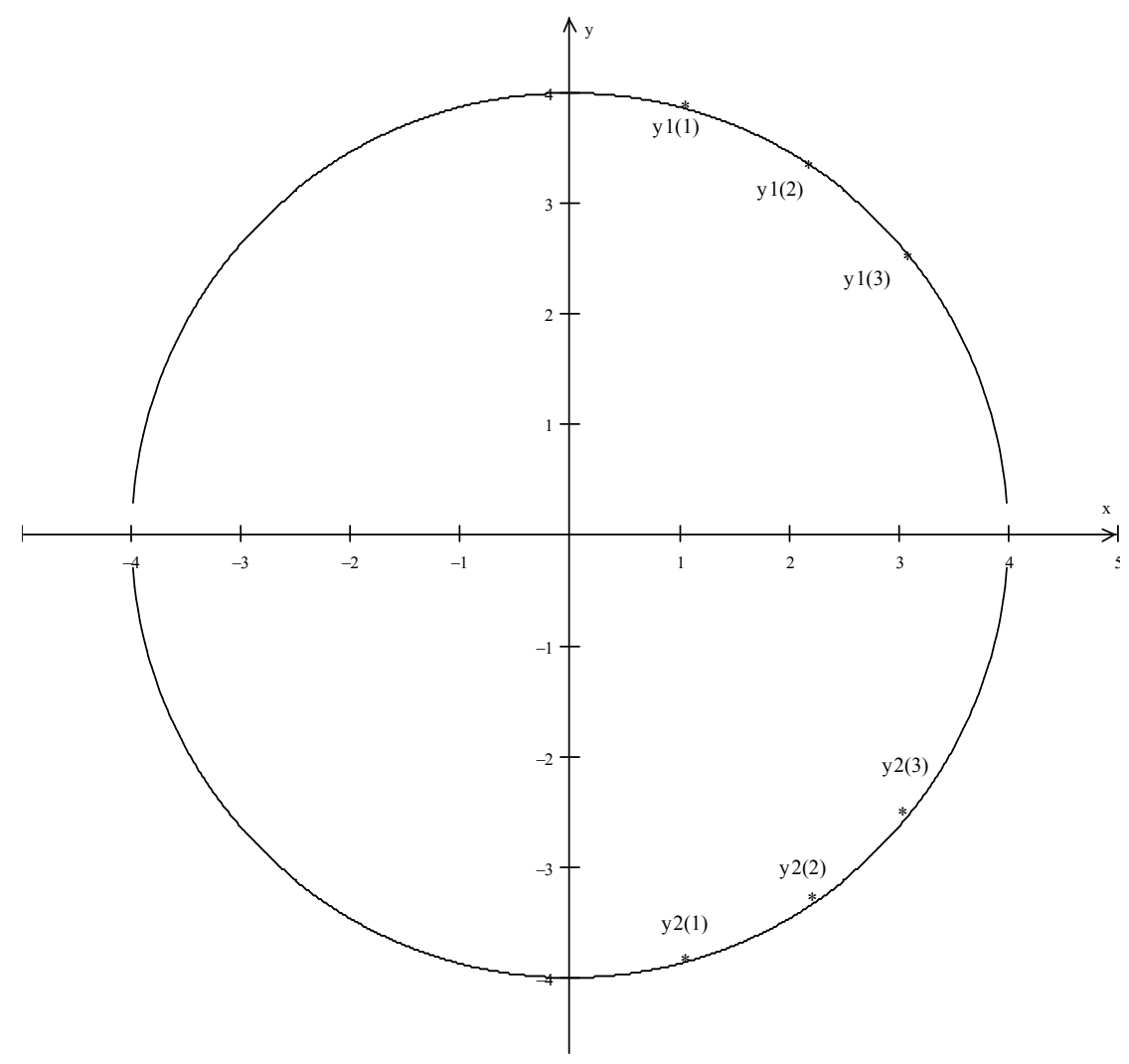

Figure 3 An example of the graph of the points on a multi-valued curve, a circle of radius $=4$.

\section{Basic Terms in Analytic Geometry - points and lines}

The position of a point in the 2-dimensional plane is determined by two co-ordinates (x, y). Conventionally $\mathrm{x}$ is plotted horizontally and $\mathrm{y}$ is plotted vertically. If two points are chosen, the difference in their vertical positions, $\mathrm{y}_{2}-\mathrm{y}_{1}$, is called the rise from the first point, $\mathrm{P}_{1}$, to a second, $\mathrm{P}_{2}$. The difference in their horizontal positions, $\mathrm{x}_{2}-\mathrm{x}_{1}$, is called the run from $\mathrm{P}_{1}$ to $\mathrm{P}_{2}$. See Figure 4.

The rise $=\mathrm{y}_{2}-\mathrm{y}_{1}$, is called the change in $\mathrm{y}$ and is represented by the symbol $\Delta \mathrm{y}$. The run $=\mathrm{x}_{2}-\mathrm{x}_{1}$, is called the change in $\mathrm{x}$ and is represented by the symbol $\Delta \mathrm{x}$.

From geometry, it is known that only one line can pass through two points. Lines which are not vertical must cross the $y$-axis at a unique point called the $\mathbf{y}$-intercept. In addition, lines which are not horizontal must cross the $\mathrm{x}$-axis at a unique point called the $\mathbf{x}$-intercept.

In the field of aviation, the direction of the flight of an aircraft is described by the angle with the north called the bearing. Another angle used in many applications is the angle of inclination, $\alpha$, which is the angle that a slanted line on the 2-dimensional, $\mathrm{x}-\mathrm{y}$ Cartesian plane makes with the positive horizontal axis. Any line has only one angle of inclination which can be determined from the ratio of the rise to run, $\Delta \mathrm{y} / \Delta \mathrm{x}$. This ratio, which is called the difference quotient, can be shown by similar triangles to be constant for any two points on a straight line. The angle of inclination determines the difference quotient and vice versa. 


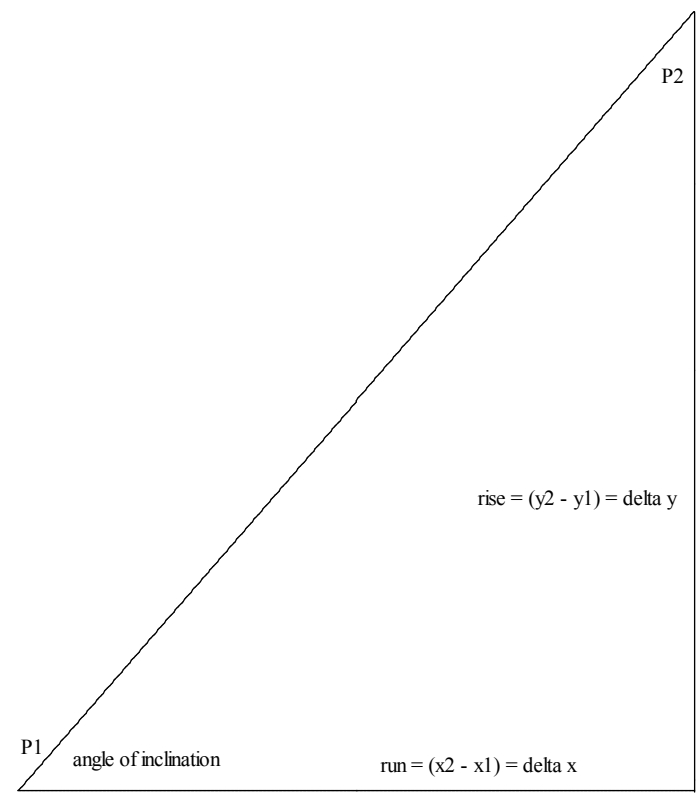

Figure 4 Rise, run and the angle of inclination

The difference quotient is a major idea in the study of straight lines. It is also a major idea in the study of the curves of calculus. Not only does the difference quotient indicate the direction of lines in the plane but it also serves as a magnifying ratio which multiplies changes in $\mathrm{x}$ to produce changes in $y$. As an example, for the line $y=3 x+2$, for any pair of points, changes in $y$ that is $\mathrm{y}_{2}-\mathrm{y}_{1}=\Delta \mathrm{y}$, equals 3 times the corresponding change in $\mathrm{x}, \mathrm{x}_{2}-\mathrm{x}_{1}=\Delta \mathrm{x}$.

\section{The Algebra of Straight Lines}

The simplest of the algebraic equations where the variables, say $\mathrm{x}$ and $\mathrm{y}$ appear only to the first degree have the form $\mathrm{Ax}+\mathrm{By}=\mathrm{C}$. As long as both $\mathrm{A}$ and $\mathrm{B}$ are not zero, the graphs of these first degree equations are straight lines. In this paper the word curve will be used to mean all graphs of algebraic equations including straight lines. Straight lines are completely determined by two properties, such as two points or one point and a direction. Every line has a unique direction and if the line is not vertical a unique position.

If both $\mathrm{A}$ and $\mathrm{B}$ are not zero and $\mathrm{C}$ is zero, the line will pass through the origin. If $\mathrm{B}$ is not zero, the equation $A x+B y=C$ can be solved for $y$ to obtain a form $y=(C / B)-(A / B) x$. Introduce the single letter $m$ to replace the expression $-(A / B)$ and the single letter $b$ to replace the value of $\mathrm{C} / \mathrm{B}$. Then the equation for a line takes the form $\mathrm{y}=\mathrm{mx}+\mathrm{b}$. The value of $\mathrm{b}$ determines the $\mathrm{y}-$ intercept or vertical position of the line and $\mathrm{m}$, which is called the slope of the line, determines the direction of the line. The form $y=m x+b$ is called the slope-intercept form of straight line. Parallel lines have the same direction and therefore must have equal slopes as shown in Figure 5 below.

If $\mathrm{m}=$ zero, the line is horizontal.

If $\mathrm{m}>$ zero, the line slants upward toward the first quadrant.

If $\mathrm{m}<$ zero, the line slants downward toward the fourth quadrant. 


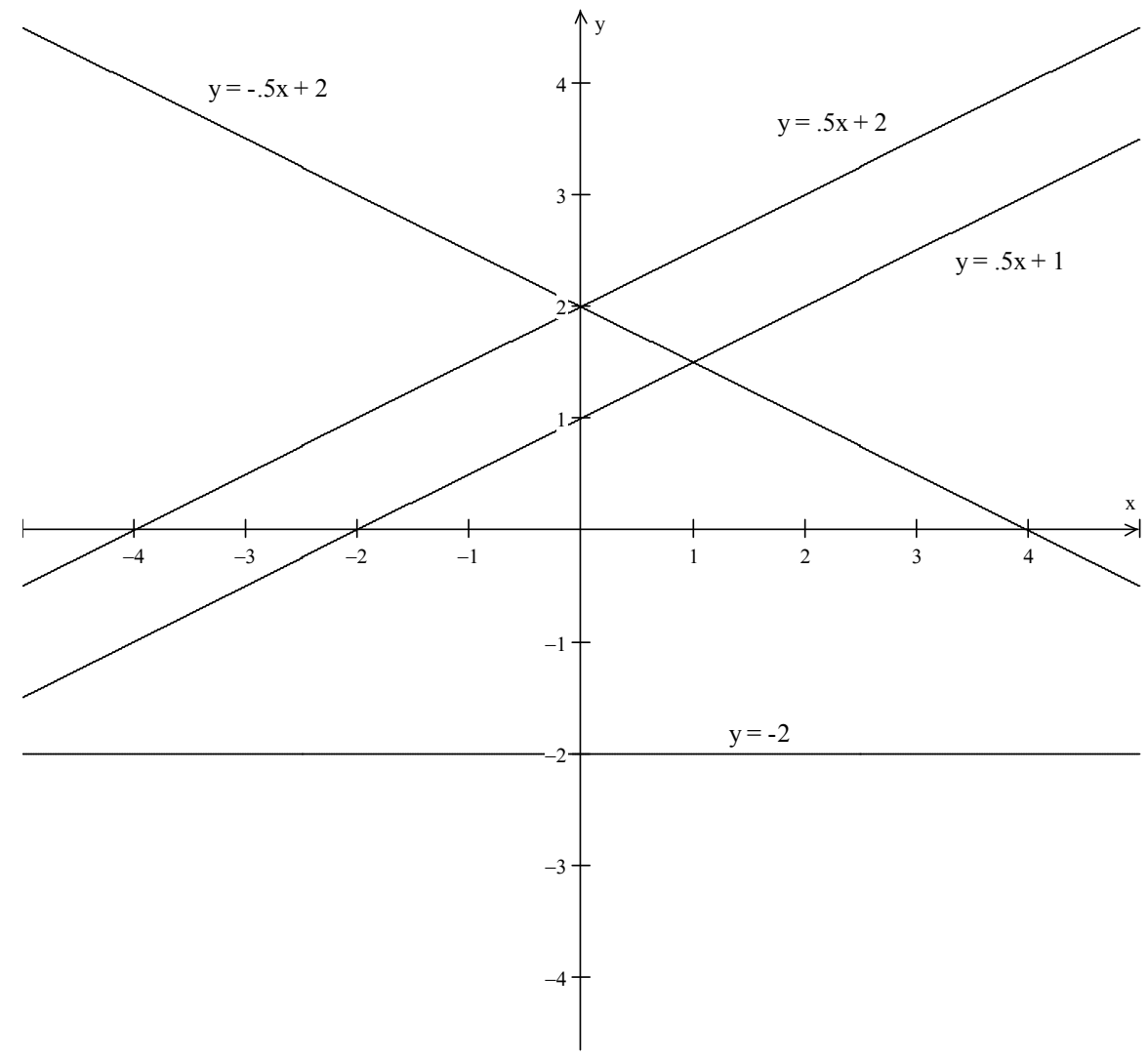

Figure 5 Examples of lines with differing positions and directions

A line with a larger slope rises faster than a line with a smaller slope as is seen in Figure 6 below.

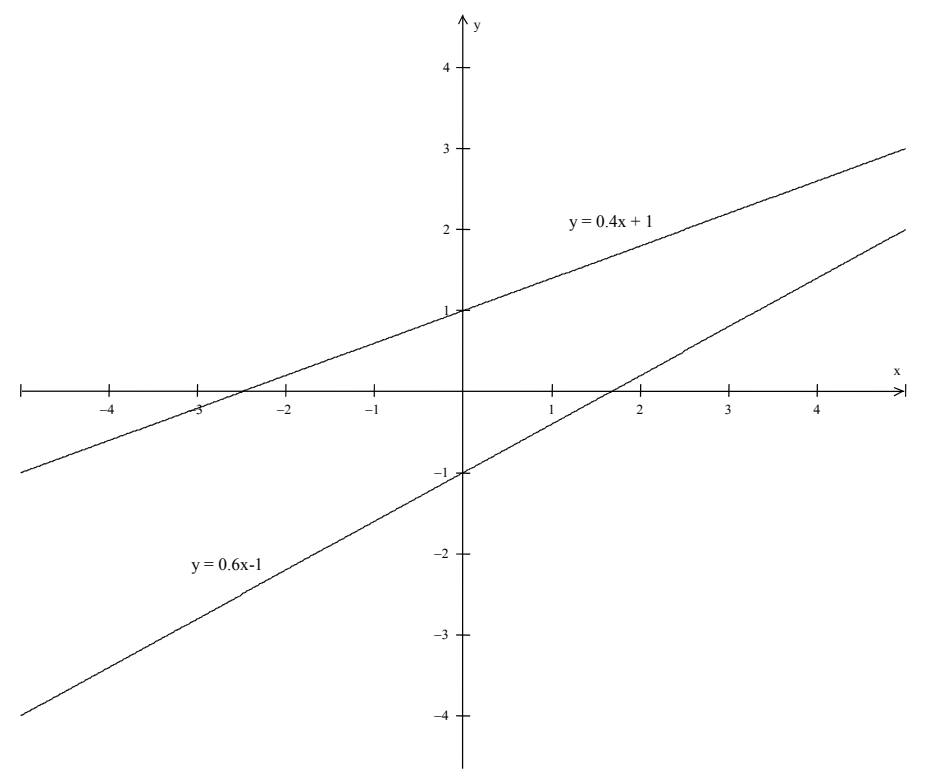

Figure 6 Examples of the directions and slopes of lines 
It is seen from the equation $\mathrm{y}=\mathrm{mx}+\mathrm{b}$ that when $\mathrm{x}$ is increased by one unit at any point on the line, the accompanying vertical rise will be increased by $m$ units. That is, for any two points whose run, $\left(\mathrm{x}_{2}-\mathrm{x}_{1}\right)$, is one, the rise, $\left(\mathrm{y}_{2}-\mathrm{y}_{1}\right)$, will be $\mathrm{m}$. In addition the difference quotient, $\Delta \mathrm{y} / \Delta \mathrm{x}$ between any pair of points is seen to be always equal to the slope, $\mathrm{m}$, of the line passing through the two points.

$$
\mathrm{m}=\left(\mathrm{y}_{2}-\mathrm{y}_{1}\right) /\left(\mathrm{x}_{2}-\mathrm{x}_{1}\right)=\Delta \mathrm{y} / \Delta \mathrm{x} \quad \text { Equation } 2
$$

In the right triangle of Figure 4 it is seen that the length of the side opposite to the angle of inclination equals the rise, $\mathrm{y}_{2}-\mathrm{y}_{1}$. The horizontal distance, $\mathrm{x}_{2}-\mathrm{x}_{1}$, the run, equals the length of the side adjacent to the angle, $\alpha$, of inclination. The ratio of the two sides (opposite over adjacent $),\left(\mathrm{y}_{2}-\mathrm{y}_{1}\right) /\left(\mathrm{x}_{2}-\mathrm{x}_{1}\right)$ is the definition of the tangent of the angle, $\alpha$. A scientific calculator can be used to compute the slope, $\mathrm{m}$, of a straight line from a given angle, $\alpha$.

$$
\mathrm{m}=\left(\mathrm{y}_{2}-\mathrm{y}_{1}\right) /\left(\mathrm{x}_{2}-\mathrm{x}_{1}\right)=\tan (\alpha) \quad \text { Equation } 3
$$

The same equation can be solved for the angle, $\alpha$, in order to compute the angle corresponding to a given slope.

$$
\alpha=\arctan (\mathrm{m}) \quad \text { Equation } 4
$$

Equation 2 can be rewritten to obtain another form for straight lines, called the point-slope form,

$$
\left(\mathrm{y}-\mathrm{y}_{1}\right)=\mathrm{m}\left(\mathrm{x}-\mathrm{x}_{1}\right) \quad \text { Equation } 5
$$

Equation 5 states that the vertical change, $\left(y-y_{1}\right)$, between any variable point on the line and a fixed point on the line is always $\mathrm{m}$ times the horizontal change, $\left(\mathrm{x}-\mathrm{x}_{1}\right)$. This form provides a way to obtain the equation of a line when any point, $\mathrm{P}_{1}\left(\mathrm{x}_{1}, \mathrm{y}_{1}\right)$ on the line and the slope, $\mathrm{m}$, of the line are given.

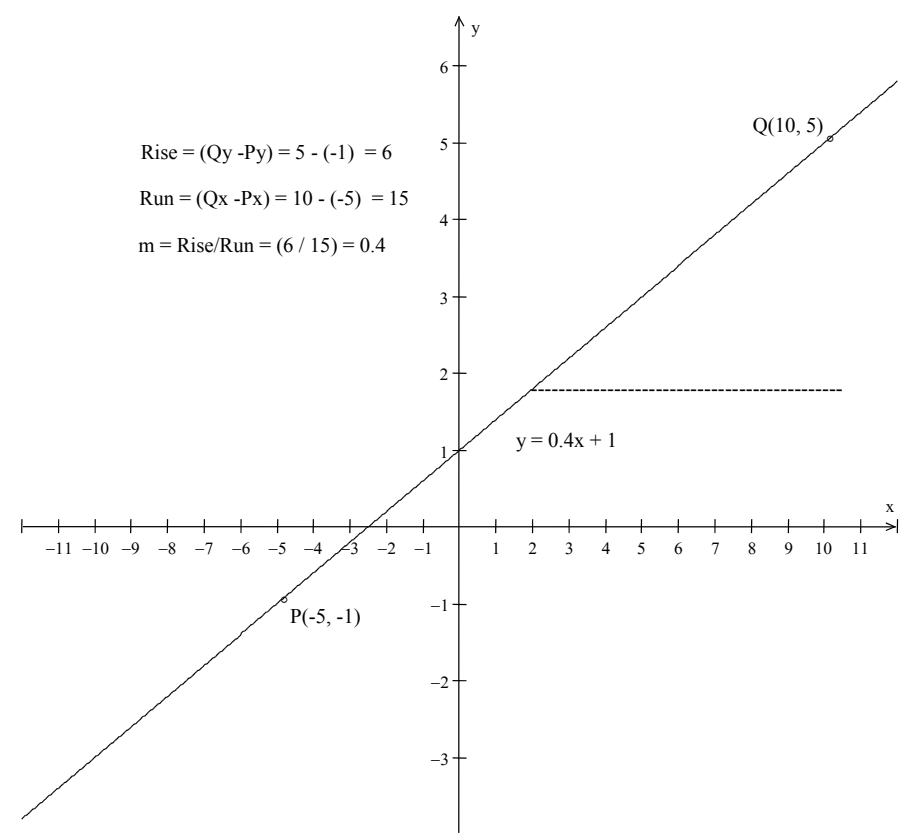

Figure 7 The slope, $\mathrm{m}$, of a line 
If the horizontal variable is time, the slope is said to represent the rate of change of the variable $y$. If the equation represents the linear motion of a particle, then the graph will display the position of the particle plotted vertically, at each instant of time, which is plotted horizontally. The slope of the line will be the change in distance divided by the change in time which is the speed of the particle.

In review, the position and direction are the two most important characteristics of straight lines. The position of a line can be described by its y-intercept, $\mathrm{b}$ or its $\mathrm{x}$-intercept, and its direction is described by its slope, $\mathrm{m}$. As can be seen in Figure 5, increasing the y-intercept, that is, raising the line without changing the slope will affect the $\mathrm{x}$-intercept. Since the direction of straight lines is constant everywhere, straight lines have no curvature.

\section{Analytic Geometry vs. Pre-calculus}

During the decade or so following World War II, it was common for engineering students to begin their study of mathematics by taking a course called "Analytic Geometry \& Calculus," sometimes using a separate textbook called Analytic Geometry. Pre-calculus was not yet in vogue. The primary idea was that algebraic equations could be associated with a curve that could be graphed. This part of the course consisted of becoming familiar with the equations and their curves and the properties of these curves. Consider whether the best way to introduce the concepts of the calculus course to non-math majors is to emphasize the curves of analytic geometry or the functions of pre-calculus.

The discussion which follows concerns equations in two variables, $\mathrm{x}$ and $\mathrm{y}$. The graphs of these equations will plot $\mathrm{x}$ along the horizontal axis and $\mathrm{y}$ along the vertical. When an algebraic equation has more than one $\mathrm{y}$-value for a particular $\mathrm{x}$ over an interval containing $\mathrm{x}$, the curve of the equation can be broken up into connected pieces over the interval called branches. An elementary example would be the graph of the equation of a circle as shown in Figure 3.

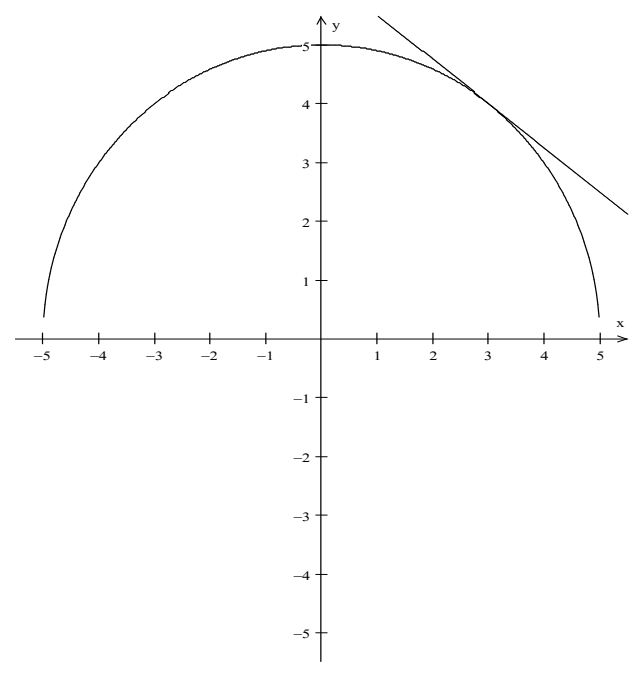

Figure 8 Upper branch of a circle

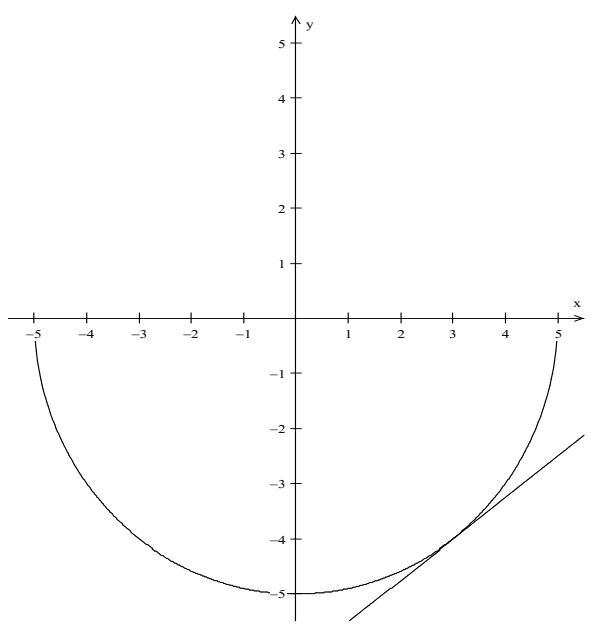

Figure 9 Lower branch of a circle 
In figures 8 and 9 it is seen that the circle does not extend beyond a distance equal to one radius from the center. For all $\mathrm{x}$-values within a distance of one radius from the center, there are two branches; one above the horizontal diameter and the other below. On a single branch, there will be one $y$-value and one tangent line for each $\mathrm{x}$. The slope of the tangent line at any point determines the direction of the curve at that point. Specifically, for a circle, centered at the origin, on the upper branch for every $x$-value, one can see that

1) the y-values are positive,

2) in the first quadrant the tangent lines slope downward,

3 ) in the second quadrant the tangent lines slope upward and

4) at each point the arc of the circle lays below the corresponding tangent line, that is, the circle is turning downward.

Furthermore; for the same circle, centered at the origin, on the lower branch for every $x$-value, it is seen that

1) the y-values are negative,

2) in the third quadrant the tangent lines slope downward,

3 ) in the fourth quadrant the tangent lines slope upward and

4) at each point the arc of the circle lays above the corresponding tangent line, that is, the circle is turning upward.

The algebraic functions of pre-calculus and elementary calculus can be considered as the branches of the algebraic curves which correspond to algebraic equations in two variables. From this point forward I will call the course that immediately precedes calculus as pre-calculus although I am convinced that the concepts that are being introduced should be based on algebraic curves. Additionally from this point forth, when the reader sees the word function, a singlevalued curve perhaps with a few jumps and corners can be visualized. The word function will be used to mean the algebraic expression which corresponds to a single-valued branch of the curve.

\section{Classes of Equations}

Equations in calculus are classified by the operations and expressions that appear in the equation. Our interest is in equations in two variables which will be called $\mathrm{x}$ and $\mathrm{y}$.

Algebraic equations in two variables are equations that can be written in a form relating sums and differences of terms which are products of numbers and powers of the variables. For examples, see equations 7, 8 and 9 in Table 1 below. Equation 6 in Table 1 shows a transcendental or non-algebraic equation which cannot be written in such a form. 
transcendental (non-algebraic) equation

$4^{\text {nd }}$ degree algebraic equation

$3^{\text {rd }}$ degree algebraic equation

$2^{\text {nd }}$ degree algebraic equation rational function

Polynomial function

$$
\begin{aligned}
& x y^{2}=2 e^{x}+1 \\
& y^{2}=x^{2}\left(1-x^{2}\right) \\
& x y^{2}=2 x^{2}+1 \\
& x y=2 x^{2}+1 \\
& y=2 x+1 / x \\
& y=x^{2}+1
\end{aligned}
$$

Equation 6

Equation 7

Equation 8

Equation 9

functional form of equation 9

Equation 10

Table 1

If in every term that y appears, as in equation $9, \mathrm{y}$ occurs only to first power possibly multiplied by powers of $\mathrm{x}$ and the other terms contain only powers of $\mathrm{x}$, then the equation can be solved for $\mathrm{y}$ to obtain a form which is a quotient of two polynomials in $\mathrm{x}$,

$$
\mathrm{y}=\mathrm{P}_{\text {num }}(\mathrm{x}) / \mathrm{P}_{\text {den }}(\mathrm{x})=\mathrm{R}(\mathrm{x}) \quad \text { Equation } 11
$$

and $\mathrm{y}$ is said to be a rational function of $\mathrm{x}$.

If in addition to y appearing only to the first power in some terms, y does not multiply any power of $x$ and the other terms contain only powers of $x$ 's and constants as in equation 10 in Table 1 then the equation can be solved for $\mathrm{y}$ to obtain a form $\mathrm{y}=\mathrm{P}(\mathrm{x})$ and in this case, $\mathrm{y}$ is said to be a polynomial function of $\mathrm{x}$. Therefore, polynomial functions can be expressed in a form comprised only of sums and differences of terms consisting of numbers multiplied by whole number powers of $\mathrm{x}$. Polynomial functions do not allow for divisions by $\mathrm{x}$ and additionally are defined and single valued everywhere.

On the other hand, rational functions are capable of permitting divisions by the variable $\mathrm{x}$ and whole number powers of the variable $\mathrm{x}$. Furthermore, rational functions are defined everywhere except at those $\mathrm{x}$-values which are zeros of the denominator polynomial. Rational functions are single valued wherever they are defined. In the explicit functional forms of an equation, $y$ alone appears to the left of the equal sign and only operations on $\mathrm{x}$ appear on the right. If the operations are single valued, then there will only be one value of $\mathrm{y}$ for each value of $\mathrm{x}$.

However for the next class of equations, the general implicit algebraic equations for example, the equation of a circle $x^{2}+y^{2}=25$, the curves can be multivalued, and there can exist intervals of $x$, called excluded intervals, for which there are no values for $y$. One mechanism of producing excluded intervals is to take the square root of function which has negative values in an interval. As for the example of the circle, the square root of the polynomial, $y=25-x^{2}$, is excluded for all $|\mathrm{x}|>5$ and double-valued for all $\mathrm{x}$ in the interval $|\mathrm{x}|<5$.

For polynomial and rational functions when the value of $y$ is not constant, then if the value of $x$ should be changed, even slightly, the value of $\mathrm{y}$ will also change. It could be said that $\mathrm{x}$ controls $y$. Call $x$ the controlling variable and $y$ the controlled variable. Call $x$ the master variable and $\mathrm{y}$ the slave variable. Conventional texts refer to $\mathrm{x}$ as the independent variable, meaning the value of $\mathrm{x}$ can be selected, and $\mathrm{y}$ is called the dependent variable, meaning the value of $\mathrm{y}$ gets controlled. However, it is possible for an equation to relate variables which trend together with 
neither controlling the other. When the independent variable is time, then the equation may describe the evolution of a process or it may describe an electronic signal.

For algebraic equations in two variables, if a value is selected for $\mathrm{x}$, then a polynomial equation remains which places a condition on the value of $y$. If the degree of this polynomial equation is $\mathrm{n}$, then for each value of $\mathrm{x}$, there can be at most $\mathrm{n}$ values of $\mathrm{y}$. Each of these $\mathrm{n}$ possible values will lie on a different branch of the algebraic curve. Consider as an example the fourth degree algebraic curve, $x^{2}=y^{2}\left(4-y^{2}\right)$ which is shown below in Figure 10. For each $\mathrm{x}$ in the interval $|\mathrm{x}|<1$, there are four branches.

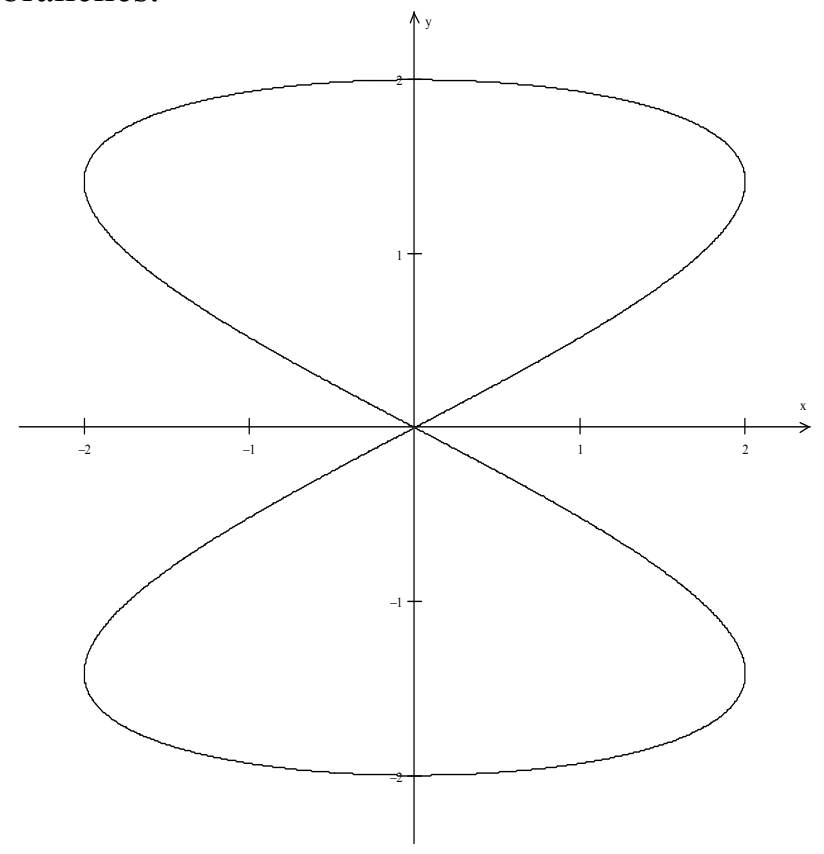

Figure 10 The curve of the equation $x^{2}=y^{2}\left(4-y^{2}\right)$

Equations, which contain non-algebraic functions such as the trig, exponential and log functions, transcend algebra and accordingly, are called transcendental equations.

\section{Algebraic Curves}

The study of the curves corresponding to algebraic equations in two variables starts in algebra or pre-calculus courses. In general, the curves naturally loop or double back on themselves, a property of curves called multiple-valued. When this phenomenon occurs, more than one value for $\mathrm{y}$ corresponds to each $\mathrm{x}$. If the curve is not multiple-valued, that is, the curve does not loop or double back, $\mathrm{y}$ is said to be a function of $\mathrm{x}$ and written as $\mathrm{y}=\mathrm{f}(\mathrm{x})$. If algebraic curves do double back like the circle or the curve shown in figure 3, the curve can be cut into continuous single-valued pieces called branches. Since on each branch there is only one value of y for each $\mathrm{x}$, the values of $\mathrm{y}$ can be considered functions of $\mathrm{x}$. So if one considers only the upper half of a circle, $y$ will be functionally related to $x$ with the equation, $y_{1}=\operatorname{sqrt}\left(25-x^{2}\right)$. On the lower branch of the circle the $y$-values will be related to $x$ by the function $y_{2}=-\operatorname{sqrt}\left(25-x^{2}\right)$. 
Moreover, the student should understand that there are advantages to working with the complete algebraic curve rather than working with two or more functional forms. A valuable solution on the lower half of a circle might be overlooked and missed if a student uses only the functional form of the upper half. The complete algebraic curve is natural and corresponds to the algebraic equation from which it was derived. However, the advantage of the functional form is that for any $\mathrm{x}$, on each branch there will be only one y-value, only one direction and only one rate of turning.

An important characteristic of an algebraic equation is its degree, which is the largest of the sums of the power of $x$ and the power of $y$ in each of its terms. It is also important not to confuse the concept of the degree of an algebraic equation with the concept of the degree of a polynomial.

The equation $x^{2} y^{3}+2 x y^{2}+3 x y^{5}=10$ has algebraic degree equal to 6 (the sum of the powers of $\mathrm{x}$ and $\mathrm{y}$ in the third term on the left) but will be a second degree polynomial in $\mathrm{x}$ for each $\mathrm{y}$ and be a fifth degree polynomial in $\mathrm{y}$ for each $\mathrm{x}$. The following principles provide some insight into the meaning of the degree of an equation:

1) A straight line can cross the curve of an $n^{\text {th }}$ degree polynomial at most $n$ times.

2) If $\mathrm{n}$ is not equal to zero, an $\mathrm{n}^{\text {th }}$ degree polynomial can be considered a special case of an $\mathrm{n}^{\text {th }}$ degree algebraic curve

3) A straight line can cross an $m^{\text {th }}$ degree algebraic curve at most $m$ times.

4) If an algebraic curve is $m^{\text {th }}$ degree in $y$, there can be no more than $m$ branches over any value of $x$.

5) If an algebraic curve is $n^{\text {th }}$ degree in $x$, there can be no more than $n$ intersections with a horizontal line.

\section{Transcendental equations and their curves}

The student who has passed pre-calculus is familiar with the everywhere defined, single-valued continuous and oscillating sine curve. The sine curve does not correspond to a polynomial, or an algebraic equation. An infinite number of angles, $\Theta$, for which $\sin (\Theta)=1 / 2$, means that there are an infinite number of crossings of the sine curve with the line $y=1 / 2$. The angles, $\Theta$, for which $\sin (\Theta)=1 / 2$, that is, the curve of $y=\operatorname{arc} \sin (\Theta)$ has an infinite number of branches. If the interval of $\Theta$ is restricted to one branch $-\pi / 2<\Theta<\pi / 2$, then the equation $y=\operatorname{arc} \sin (\Theta)$ can be seen as a single valued function of $\Theta$, meaning that for each value of $\Theta$ in the interval $-\pi / 2<\Theta<\pi / 2$ there will be only one value for $y$ and one tangent line to the curve.

The following properties of the transcendental curves can be observed by examining their graphs. The oscillating sine and cosine curves are defined everywhere and single-valued but bounded above and below by their peak values. The exponential curves are also single-valued and defined everywhere. The logarithmic curves are single-valued but defined only for positive values of $x$. The functions $\tan (\Theta)$, and $\sec (\Theta)$ are single valued where they are defined but are undefined at an infinite number of isolated points where $\cos (\Theta)=0$, i.e. the odd multiples of $\pm \pi / 2$. Similarly the functions $\cot (\Theta)$, and $\csc (\Theta)$ are also single valued where they are defined 
but are also undefined at the infinite number of isolated points where $\sin (\Theta)=0$, i.e. the whole number multiples of $\pi$.

\section{Limits}

Among the important features of algebraic curves are continuity, point gaps, jumps, slopes of tangent lines and cusps. How should these features, which conventionally follow discussions of limits and delta-epsilon arguments, be introduced? As properties of curves, these concepts can be introduced intuitively. If a point is removed from a curve, there will be a gap and the curve will be discontinuous. If a step is added to a branch of a curve, then the branch will have a jump and be discontinuous. Polynomials are continuous everywhere and, therefore to find the limit at any point, simply evaluate the polynomial. The curves of rational functions are continuous except at the points where the denominator has zeros, the singular points.

1) To find the limits of rational curves at points which are not singularities, simply evaluate the rational functions.

2) If the denominator has factors at points where there are no corresponding numerator factors, then an evaluation will produce a form of 'some numerator value over zero'. In this case, the curve has a vertical asymptote at that point, a feature that electrical engineers and mathematicians in advanced courses call a pole. The vertical values for values of $\mathrm{x}$ near the pole are unbounded. To provide a more fluent description, this case is described by saying that the curve extends to infinity.

If the singularities are of the form 'zero over zero', then these zeros are produced by common numerator and denominator factors.

3) If the number of zeros of the denominator at a point exceeds the number of zeros of the numerator at that point, then the curve has a pole.

4) However, if the number of zeros of the denominator at a point is less than the number of zeros of the numerator at that point, then the excess factors in the numerator, and in the denominator can be cancelled. An evaluation of the function with the excess factors removed will yield the value zero, that is, at that point, the limit is zero.

5) Lastly, if the number of numerator factors and denominator factors are equal, then these factors can be cancelled, and the singularity will be removed. Evaluating the remaining function will yield the limit of the function at the singularity.

The preceding approach can convey the concept of the limit without the intricacies of deltaepsilon arguments. It is more important for the general public to understand that performing the four basic operations of addition, subtraction, multiplication and division of continuous and smooth branches of curves will yield continuous and smooth curves, except when a zero occurs in the denominator. More can be found concerning limits in my paper ${ }^{6}$ on mathematical definitions. 


\section{Pre-calculus summary}

The strategy is clear. There is a strong correspondence between algebraic equations in two variables and algebraic curves. We can be using this natural correspondence to define the concepts that engineering and technology students will be studying in their beginning calculus courses. If the functions of calculus are defined as the single-valued branches of curves, then many of the concepts of calculus are given meaning as features of these curves - intercepts, symmetries, limits, continuity, tangent lines, slope, concavity, etc. Polynomials and rational functions are automatically single valued. Additionally, the other functions studied in the calculus course are branches of algebraic curves and elementary transcendental equations. There can be no doubt that the single valued continuous branch of a curve is a clear concept which will provide focus in the study of calculus. If the definition of a function is taken to be the algebraic expression which corresponds to the branch, there is no conflict with the conventional definition of a function as ordinarily used in calculus.

As was asserted in the abstract, Pre-calculus is a much more difficult subject for a student to master than is calculus. The concepts involved in manipulating equations and the various form changing operations on equations such as factoring, completing the square and solving are unfamiliar and new to a beginning student. In addition the student is required after a glance at an equation to decide what is needed and be able to perform an appropriate calculation.

After completing a pre-calculus course, a student should recognize when there is a need and be able:

1) to factor and solve simple polynomials,

2) to verify identities of polynomial and rational functions,

3) to graph polynomials, rational functions and compositions of these functions,

4) to "complete the square" in order to find properties of conic sections,

5) to compute using negative and fractional exponent notation,

6) to graph trig functions, verify trig identities,

7) to solve simple trig conditional equations,

8) to graph simple exponential and log functions, verify their identities and

9) to solve simple exponential and logarithmic conditional equations.

Additionally, the student should have a visual comprehension of all the technical words that are needed to describe the important features of functions and curves: inverse forms, the symmetries, monotonic, continuity, differentiability, etc.

The classic curves of mathematics provide a wonderful means to introduce students to the concerns of calculus. A student can ponder derivative properties of the curves including the slopes of tangent lines, concavity and the points of inflection. The student can ponder the integral properties of the curves including areas and arc lengths. A young student might want to study calculus in order to learn how the formulas for the circumference and area of a circle were derived. A student might want to compare the circumference and area of the astroid to that of a circle. To attract more students to the study of calculus, start with the concepts of mainstream continuous, smooth curves and postpone the obstacles presented by singularities. 
Many of the explanations that can be found on the Internet repeat presentations provided decades ago which led to and were part of the now considered failed "New Math" program. However, the Internet can be a valuable aid in the study of curves. Animations of the trajectories of moving points such as the astroid and cycloid can be found on the Internet,. To assist in the study, free plotting software such as WINPLOT ${ }^{13}$ can be downloaded to make obtaining computer solutions almost effortless, and these computer solutions provide a check on the student's analytic calculations. The website www.calc101.com will perform polynomial multiplications, polynomial divisions and step by step partial fraction expansions.

Partial fraction expansions appropriately belong in a pre-calculus course as a form changing operation on rational functions. Instead, students may first encounter these expansions near the end of an integral calculus course where the form changes are tactics in the integration of rational functions. Collections of convenient mathematical formulae can be found on the website ${ }^{12}$ maintained by Paul Dawkins.

This completes part 1, which is intended to serve as an introduction to the correspondence between algebraic curves and equations. In part 2 , the concepts of derivatives and concavity will be discussed visually and strategically. A student who feels at ease with the above tasks and concepts will find after, reading part 2 on differential calculus, that there are only a few more new concepts. The student could then concentrate on mastering the few differentiation rules and acquiring dexterity performing differentiations.

\section{References}

1. Aleksandrov, et al Mathematics Its Content, Methods and Meaning

The MIT Press, Cambridge MA, 1963

2. Alsina, Claudi \& Nelson, Roger B. Math Made Visual MAA, Washington, 2006

3. Grossfield, Andrew What are Differential Equations? A Review of Curve Families ASEE Annual Conference, 1997

4. Grossfield, Andrew The Natural Structure of Algebra and Calculus ASEE Annual Conference, 2010

5. Grossfield, Andrew Mathematical Forms and Strategies ASEE Annual Conference, 1999

6. Grossfield, Andrew Mathematical Definitions: What is this thing? ASEE Annual Conference, 2000

7. Grossfield, Andrew Are Functions Real? ASEE Annual Conference, 2005

8. Grossfield, Andrew Wonder, Discovery and Intuition in Elementary Mathematics Proceedings of the ASEE Zone 1 Conference at West Point, 2008

9. Grossfield, Andrew Visual Analysis and the Composition of Functions ASEE Annual Conference, 2009

10. Nelson, Roger B. Proofs without Words: Exercises in Visual Thinking MAA Washington, 1993

11. Nelson, Roger B. Proofs without Words II: More Exercises in Visual Thinking MAA Washington, 2000

12. Dawkins, Paul http://tutorial.math.lamar.edu

13. Parris, Rick WINPLOT, a general-purpose plotting utility, Peanut Software 